\title{
Evaluation of a GCM cirrus parameterization using satellite observations
}

\author{
B. J. Soden ${ }^{1}$ \\ Department of the Geophysical Sciences, University of Chicago, Illinois \\ L. J. Donner \\ NOAA Geophysical Fluid Dynamics Laboratory, Princeton University, Princeton, New Jersey
}

\begin{abstract}
This study applies a simple yet effective methodology to validate a general circulation model parameterization of cirrus ice water path. The methodology combines large-scale dynamic and thermodynamic fields from operational analyses with prescribed occurrence of cirrus clouds from satellite observations to simulate a global distribution of ice water path. The predicted cloud properties are then compared with the corresponding satellite measurements of visible optical depth and infrared cloud emissivity to evaluate the reliability of the parameterization. This methodology enables the validation to focus strictly on the water loading side of the parameterization by eliminating uncertainties involved in predicting the occurrence of cirrus internally within the parameterization. Overall the parameterization performs remarkably well in capturing the observed spatial patterns of cirrus optical properties. Spatial correlations between the observed and the predicted optical depths are typically greater than 0.7 for the tropics and northern hemisphere midlatitudes. The good spatial agreement largely stems from the strong dependence of the ice water path upon the temperature of the environment in which the clouds form. Poorer correlations $(r \sim 0.3)$ are noted over the southern hemisphere midlatitudes, suggesting that additional processes not accounted for by the parameterization may be important there. Quantitative evaluation of the parameterization is hindered by the present uncertainty in the size distribution of cirrus ice particles. Consequently, it is difficult to determine if discrepancies between the observed and the predicted optical properties are attributable to errors in the parameterized ice water path or to geographic variations in effective radii.
\end{abstract}

\section{Introduction}

The importance of clouds in regulating the Earth's radiative energy balance is widely recognized. However, despite this importance, a clear understanding of the interactions among clouds, radiation, and climate has not been achieved. Numerous theoretical studies have demonstrated the sensitivity of general circulation model (GCM) simulations to the treatment of cloud-radiative interactions [Shukla and Sud, 1981; Ramanathan et al., 1983; Randall et al., 1989; Cess et al., 1990]. Indeed, cloud-radiative processes are presently recognized as the single largest known source of uncertainty in GCM predictions of the potential climate change resulting from increasing $\mathrm{CO}_{2}$. Because of their cold radiating temperatures, cirrus clouds have a dramatic impact on the outgoing longwave radiation and therefore are of crucial importance in determining the radiative energy balance of the planet. The decisive role which cloud-radiative interactions play in determining climate sensitivity and the importance of cirrus clouds in regulating the radiative energy balance motivate the present study.

\footnotetext{
${ }^{1}$ Now at Atmospheric and Oceanic Sciences Program, Princeton University, Princeton, New Jersey.

Copyright 1994 by the American Geophysical Union.

Paper number 94JD00963.

0148-0227/94/94JD-00963\$05.00
}

Much of the difficulty in modeling cloud-climate feedback stems from the problem of predicting the optical properties of clouds based upon characteristics of the large-scale flow in which they occur. The dynamic and thermodynamic variables describing the large-scale flow are predicted explicitly in a GCM; however, the characteristics of the cloud field can exhibit spatial variations at scales several orders of magnitude smaller than that of a typical GCM grid box. Therefore it is necessary to parameterize the cloud optical properties as functions of the explicitly resolved variables in order to incorporate their effects into GCMs. Recently, Heymsfield and Donner [1990], hereafter referred to as HD, described a parameterization which predicts the ice content of cirrus associated with large-scale vertical lifting as a function of the corresponding grid-scale flow. This study combines satellite observations of cloud optical properties with European Centre for Medium-Range Weather Forecasts (ECMWF) global analyses of the temperature and wind to evaluate the ability of the HD parameterization to predict the ice water path of cirrus clouds. The approach of this study differs from that of previous investigations [Kiehl and Ramanathan, 1989; Smith and Vonder Haar, 1991; Soden, 1992] in which satellite observations were used to evaluate GCM simulations of the radiative effects of clouds in the following respects:

1. This study focuses strictly on the parameterization of large-scale cirrus clouds. 
2. ECMWF operational analyses are utilized rather than GCM simulations in an attempt to provide reliable input variables for the parameterization and reduce errors in the simulated cloud properties due to deficiencies in the input parameters.

3. The contemporaneous input variables and satellite observations enable the occurrence of cirrus clouds within a given analysis grid box to be prescribed, while the ice water content is calculated based upon the corresponding dynamic and thermodynamic variables for that grid box. This eliminates the uncertainty of predicting the occurrence of the cirrus clouds internally within the parameterization and enables the evaluation to focus strictly on the parameterization of cirrus optical properties. Finally, although this study investigates a specific cloud parameterization, it also serves the more general purpose of illustrating a methodology for combining operational analyses and satellite observations to evaluate a variety of physical parameterizations (e.g., cloud, convective, precipitation).

Specifically, this study simulates the distribution of the vertically integrated ice water content, or ice water path (IWP), using the ice water content parameterization of HD and the corresponding cloud thickness parameterization. Section 2 describes the ice water content, cloud thickness, and optical depth parameterizations. A discussion of the model and satellite data utilized in this study is provided in section 3. In section 4 the parameterized IWP is compared with satellite measurements of visible optical depth and effective cloud emissivity, and the physical mechanisms responsible for the agreement between the parameterizations and the observations are discussed. Finally, the results of this study are recapitulated in section 5 .

\section{Parameterization of Cirrus Optical Properties}

\subsection{Calculation of Ice Water Path}

This study evaluates the GCM parameterization of ice water content developed by Heymsfield and Donner [1990]. The parameterization predicts the vertically averaged ice water content ( $\overline{\mathrm{IWC}}$ ) of cirrus clouds associated with largescale vertical lifting as a function of the resolved dynamic and thermodynamic state of the atmosphere. The IWC is calculated by reconstructing the parcel trajectory in largescale flows in which cirrus clouds are presumed to occur and represents an equilibrium between ice production due to vertical lifting and ice depletion due to gravitational settling,

$$
\frac{d X_{s}}{d t}=\dot{D}-\dot{S}=0
$$

where $X_{s}$ is the ice mass mixing ratio $\left(X_{s}=\overline{\mathrm{IWC}} / \rho\right), \rho$ is the density of air, $\dot{D}$ is the rate of deposition, and $\dot{S}$ is the rate of sedimentation. The parameterization, described in detail by HD, predicts the $\overline{\mathrm{IWC}}$ as a function of the temperature $(T)$, lapse rate $(\Gamma)$, vertical velocity $(w)$, and atmospheric pressure $(p)$ for the layer in which the cloud forms. Explicit solution of the parameterization typically requires numerous iterations, as outlined in Table 1 of HD. However, this procedure is too computationally burdensome for realistic application in GCMs. To ameliorate this problem, a lookup table based upon a set of a priori calculations for selected values of $T, p, w$, and $\Gamma$ was constructed. The IWP is then determined according to IWP $=\int_{z_{1}}^{z_{2}}$ IWC $(z) d z=\overline{\text { IWC }}$ $\Delta z_{\text {cld }}$. The calculation of cirrus cloud thickness $\left(\Delta z_{\text {cld }}\right)$ follows that of Donner [1994]. In the HD parameterization the rate of sedimentation $(\dot{S})$ is determined by considering the local vertical gradient of ice mass flux,

$$
-\frac{1}{\rho} \frac{\partial}{\partial z}\left(\rho X_{s} \bar{V}_{t}\right)=\dot{S}
$$

where $\bar{V}_{t}$ is the mean terminal velocity for a distribution of ice particles. The gradient on the left-hand side of (2) is calculated by applying the large-scale lapse rate across a layer of the cloud interior. Since the temperature varies across the layer, deposition proceeds at different rates, resulting in an ice mass flux which varies with height. Since the average value of $X_{s}$ in the layer is representative of the entire ice cloud, the sedimentation rate must also apply for the cloud as a whole. For the entire cloud, no ice enters the top, while ice settles from the base. Consequently, the cloud geometric thickness can be determined by equating the rate of ice-mass flux out of the base of the cloud to the sedimentation rate,

$$
\Delta z_{\mathrm{cld}}=\frac{\bar{V}_{t} X_{s}}{\dot{S}}
$$

For typical parameter ranges, $\Delta z_{\text {cld }}$ varies from about $100 \mathrm{~m}$ to $3 \mathrm{~km}$. Thus ice clouds can be considerably thinner than a GCM layer. For computational efficiency the calculation of $\Delta z_{\text {cld }}$ is also performed via a lookup table using a procedure identical to that for the IWC. Further details regarding the parameterization of IWC or $\Delta z_{\text {cld }}$ can be found in the work of Heymsfield and Donner [1990] and Donner [1994], respectively.

\subsection{Calculation of Optical Depth}

The key parameter which determines the radiative properties of a cloud is the optical depth $(\tau)$ which is related to the IWP according to $\tau_{\lambda}=3 \bar{Q}_{\lambda} \mathrm{IWP} / 4 r_{e} \rho_{\text {ice }}$ where $r_{e}$ is effective radius and $\rho_{\text {Ice }}$ is the density of ice $\left(780 \mathrm{~kg} / \mathrm{m}^{3}\right)$. $\bar{Q}_{\lambda}$ is the effective extinction efficiency at wavelength $\lambda$ which is calculated via application of van de Hulst's anomalous diffraction theory [Ackerman and Stephens, 1987; Stephens et al., 1990]. For calculating visible optical depth, $\lambda=0.6 \mu \mathrm{m}$; for infrared optical depth, $\lambda=15 \mu \mathrm{m}$. Spherical ice particles with $r_{e}=10 \mu \mathrm{m}$ have been assumed in calculating all optical depths to simplify the interpretation of $\tau$ in terms of IWP and to provide consistency with the International Satellite Cloud Climatology Project (ISCCP) optical depths used to evaluate the parameterization. However, in reality, ice particles may occupy a variety of crystal habits. Recent studies [Minnis et al., 1990; Wielicki et al., 1990; Takano et al., 1992] have indicated that the treatment of ice crystals in radiative transfer models as nonspherical particles provides better agreement with observations of reflected solar radiation than spherically shaped particles. A recognized difference between the optical properties of spherical and nonspherical particles is that the asymmetry parameter, $g$, which measures the ratio of forward to backscattered radiation, is systematically smaller for nonspherical particles $(g \sim 0.7)$ than for spherical particles $(g \sim 0.9)$. Unfortunately, little is known concerning the relative frequency of various crystal habits, their size distributions, and 
the dependence of these quantities upon the environment in which the cloud forms. Indeed, estimates of the effective radius of cirrus particle size distributions quoted in recent literature vary by over an order of magnitude (e.g., 5-10 $\mu \mathrm{m}$, Prabhakara et al. [1988]; $200 \mu \mathrm{m}$, Wielicki et al. [1990]). From a cloud parameterization perspective this range of uncertainty contributes directly to an equally large range of uncertainty in determining $\tau$ from model predictions of IWP. Furthermore, in the visible spectrum the extinction efficiency for this range of size parameters is approximately 2 , for both spherically and nonspherically shaped particles [Ebert and Curry, 1992]. Thus for a given IWP the fundamental difference in $\tau$ between spherical and nonspherical ice particles is due to the differing ratios of particle mass to particle cross section for these two shapes. For nonspherical particles this is crucially dependent upon the aspect ratio of the particles and the orientation of the particles with respect to the radiation flow. Our current knowledge of these properties is preliminary at best. Hence to avoid the complications surrounding these issues and to clarify the assumptions involved in parameterizing the optical depth, spherically shaped particles are selected.

\section{Data}

The input profiles of $T, w$, and $\Gamma$ required for parameterizing the IWP are prescribed using ECMWF uninitialized analyses. This study uses the TOGA/basic level III archive for two 1-month periods: July 1-31, 1987, and January 1-31, 1988. The ECMWF forecast model is a 19-level spectral model with horizontal resolution truncated at wavenumber 106. The analysis system is a three-dimensional optimal interpolation scheme which combines model forecasts based on 6-hour prior analyses with current observations from a network of surface, radiosonde, and satellite-based platforms. The analyzed fields are assimilated 4 times per day $(0000,0600,1200,1800 \mathrm{UT})$ and transformed onto a reducedresolution ( $2.5^{\circ}$ latitude by $2.5^{\circ}$ longitude) rectangular grid. Vertically, the fields are interpolated from the model $\sigma$ levels to 11 standard pressure levels $(1000,850,700,500,400,300$, $250,200,150,100,50$ mbar) used in this study. One objective of using the ECMWF analyses as the source of the input parameters is to help provide reliable variables for the parameterization and reduce errors in the predicted cloud properties due to deficiencies in the input parameters. However, it is noted that the operational analyses over the tropics and data-sparse regions of the southern hemisphere depend heavily on the forecast model used for determining the first guess [e.g., Trenberth and Olson, 1988; Tiedtke et al., 1988]. This must be kept in mind when interpreting the performance of the parameterization over these regions.

The satellite measurements of cirrus optical depth used to evaluate the parameterization are derived from the ISCCP data set. The ISCCP platform consists of four geostationary satellites and two polar-orbiting satellites which provide near-global coverage with a temporal resolution of 3 hours. Each satellite measures the upwelling radiation in one visible $(0.6 \mu \mathrm{m})$ and one infrared $(11 \mu \mathrm{m})$ spectral channel. These measurements are combined with auxiliary information regarding the optical properties of the atmosphere and surface to estimate cloud top pressure and visible optical depth based upon an a priori set of radiative-transfer calculations. The derived cloud properties from each satellite pixel are

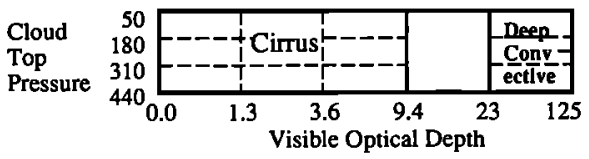

Figure 1. Cloud classification scheme based upon visible optical depth and cloud top pressure.

then averaged horizontally to an equal-area grid of roughly $(280 \mathrm{~km})^{2}$ and vertically into seven pressure intervals. To facilitate the integration with other data sets, the ISCCP data are then replicated from the equal-area grid to an equal-angle $2.5^{\circ}$ by $2.5^{\circ}$ latitude-longitude grid. For each grid box and pressure level, the number of cloudy pixels in each of five optical depth and seven cloud-top pressure categories are reported. Only the three highest cloud top pressure categories are used in this study (see Figure 1). For this study, cirrus clouds are defined by those pixels for which the visible optical depth is between 0.02 and 9.38 and the cloud top pressure is between 5 and 440 mbar. This yields nine cirrus categories. The central optical depth $\left(\tau_{i}\right)$ and central cloud top pressure $\left(p_{i}\right)$ for each cirrus category $(i)$ are then weighted by the number of pixels $\left(N_{i}\right)$ in that category to estimate a cirrus optical depth $(\tau)$ and cloud top pressure $\left(p_{\text {cld }}\right)$ for each day:

$$
\begin{aligned}
\tau & =\sum_{i} N_{i} \tau_{i} / \sum_{i} N_{i} \\
p_{\text {cld }} & =\sum_{i} N_{i} p_{i} / \sum_{i} N_{i}
\end{aligned}
$$

However, since only the number of pixels for each optical depth category is reported (rather than the average optical depth for each category), comparison of the predicted IWP with the ISCCP observations is only meaningful for timeaveraged quantities where the central value in each optical depth category is representative of the time-averaged value [Rossow et al., 1988]. Hence the evaluations in this study will focus primarily on monthly mean quantities. Further details on the ISCCP products and their retrieval can be found in the work of Rossow et al. [1988].

It should be noted that a slight modification to the cloud optical-property retrieval algorithm is being planned in which particles comprising cold clouds $(T<\sim 260 \mathrm{~K}$ ) will be modeled as nonspherical ice crystals rather than as water spheres. This modification will not significantly affect all retrievals, only those performed under certain viewing and illumination geometries. In these situations the impact of the modification upon the retrieved cloud properties is expected to be most significant for thin cirrus $(\tau<3)$, with a smaller effect on thicker cirrus $(3<\tau<10)$. Based upon studies by Wielicki et al. [1990] and Minnis et al. [1990], the following changes are expected: (1) a decrease in $\tau$ by $10-20 \%$ for cirrus with $3<\tau<10$ and a decrease in $\tau$ of up to $50 \%$ for thin cirrus $\tau<3$; (2) a decrease in cloud top pressure by 50-100 mbar. No change in the geographic pattern of cirrus optical depth is expected (W. Rossow, personal communication, 1994). Fortunately, a quantitative comparison is not emphasized in this paper, due to uncertainty surrounding the effective radius of cirrus particles. Rather, the evaluation 


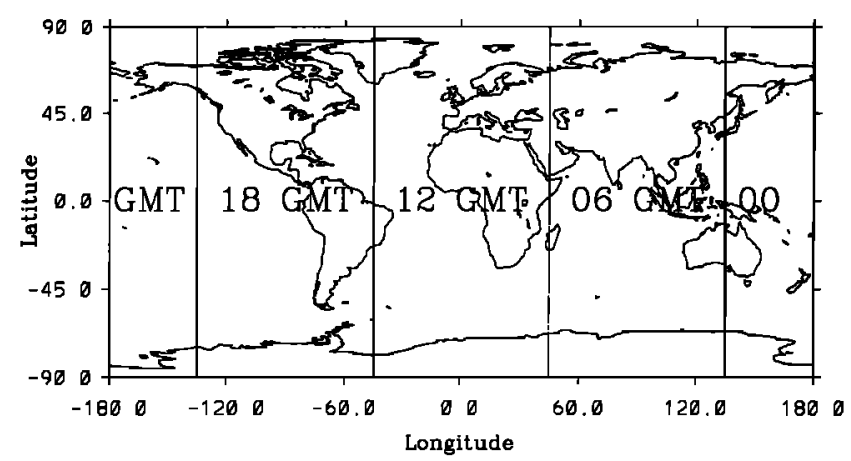

Figure 2. Map depicting the merging of the 0000,0600 , 1200 , and 1800 UT analysis grids onto a single grid.

focuses primarily on comparing the geographic patterns of cirrus optical thickness and their relation to atmospheric parameters. Since these features are not sensitive to changes in the ISCCP retrieval algorithm, the proposed modification should not affect the results of this study.

\section{Evaluation of the Parameterization}

\subsection{Procedure}

The objective of this section is to evaluate the ability of the parameterization to predict the IWP for large-scale cirrus clouds. Since the HD parameterization is designed for calculating the IWP rather than predicting the occurrence of cirrus, ISCCP observations are utilized to identify grid boxes which contain cirrus clouds as a means of prescribing their occurrence for the parameterization. The identification of cirrus is based upon the daily optical depth and cloud top pressure observations from ISCCP. In this study, cirrus clouds are defined as those cloudy pixels with optical depths less than 9.4 and cloud top pressure less than 440 mbar (see Figure 1). Since the parameterization is intended to capture cirrus associated with large-scale lifting rather than cirrus resulting from deep convection, ISCCP observations are also used to exclude grid boxes containing deep-convective cloud cover, defined as clouds with optical depths greater than 22.6 and cloud top pressure less than $\mathbf{4 4 0}$ mbar. As a means of imposing these constraints, this study focuses on those $2.5^{\circ} \times 2.5^{\circ}$ grid boxes for which the cirrus cloud amount is greater than $20 \%$ and the deep-convective cloud amount is less than 5\%. Although the selection of these thresholds is somewhat arbitrary, the results of the comparison are not sensitive to their precise values. For each time, the weighted average, cloud top pressure of the cirrus pixels $p_{\text {cld }}$ is used to identify the vertical level of the cloud within each grid box. The values of $T, w, p$, and $\Gamma$ taken from the corresponding grid box and vertical level of the ECMWF analyses are input into the parameterization to compute an IWP. The predicted IWP is then transformed into an optical depth, as described in section 2, and compared with the observed cirrus optical depth from ISCCP. Results are presented in terms of monthly averages. To remove high spatial noise and focus on larger-scale patterns, both the observed and the simulated optical depths are smoothed using a 1-2-1 spatial filter in both the zonal and the meridional directions. Since both the predicted IWP and the ISCCP optical depths represent average values for the overcast portion of the grid box, the comparison procedure is insensitive to the actual amount of cirrus present. Finally, since this procedure requires estimates of the visible optical depth, comparison is only possible from daytime observations. Therefore in performing the comparison, the ISCCP observations and ECMWF analyses corresponding to 0000,0600 , 1200 , and 1800 UT for each day are merged (see Figure 2) to provide a global, daytime grid for both data sets.

\subsection{Monthly Mean Optical Depth}

Plate 1 displays the geographic distribution of the predicted (top) and ISCCP (bottom) cirrus optical depths averaged over the period July 1-31, 1987. The scale ranges from 0 (blue) to 1 (red). Maxima in both the predicted and the observed optical depth are present over the tropics, highlighting the presence of the ITCZ. Within the tropics the optically thickest clouds occur over the western half of the Pacific, west of Indonesia, and over much of Central America and the northern tip of South America. Over these areas the magnitude of the predicted optical depth is in good agreement with that observed. Maxima are also noted over central Africa, however the predicted values for these regions are as much as $25-50 \%$ lower than those observed. Minima are evident over the subtropical high-pressure systems of the southern hemisphere and parts of the northern hemisphere where few cirrus clouds occur. Secondary maxima in the cirrus optical depth occur over the midlatitudes in both the northern and the southern hemispheres. The location of these maxima closely correspond to the climatological position of the midlatitude storm tracks and are likely associated with midlatitude jet streams which play an important role in the formation of cirrus [Menzel et al., 1992]. Although the basic features are evident in both the observed and the predicted visible optical depths, the predicted values are frequently $25-50 \%$ smaller than those observed. This is particularly noticeable over the southern hemisphere midlatitudes where the monthly mean observed optical depths range from 0.4 to 0.7 , while those predicted are typically less than 0.3 .

Given the uncertainty in effective radius, the geographic differences between the observed and predicted optical depth may stem from a geographic dependence in the ice particle size distributions. The results shown here assumed an effective radius of $10 \mu \mathrm{m}$; however, present estimates of the effective radius for cirrus clouds vary by over an order of magnitude. Since the optical depth is inversely related to the effective radius, this makes quantitative validation of the parameterization difficult. Further discussion of the potential impact of spatial variations in effective radius upon the evaluation of the parameterized optical depths is given in section 4.5 .

\subsection{Conditionally Averaged Optical Depth}

Additional insight into the behavior of the parameterization and into issues surrounding its validation can be gained by examining the monthly mean optical depth averaged from only those occasions in which cirrus were present. These values are referred to as the "conditionally averaged" optical depth, since the temporal averaging is conditional upon the presence of cirrus for that time and grid box. If cirrus were not present at a given time, then that data point was not included in determining the temporal average; i.e.,

$$
\bar{\tau}=\frac{\sum_{i} \tau_{i} \delta_{i}}{\sum_{i} \delta_{i}}\left\{\begin{array}{l}
\delta_{i}=1 \text { for } \tau_{i}>0 \\
\delta_{i}=0 \text { for } \tau_{i}=0
\end{array}\right.
$$




\section{Monthly Mean Optical Depth: Predicted}

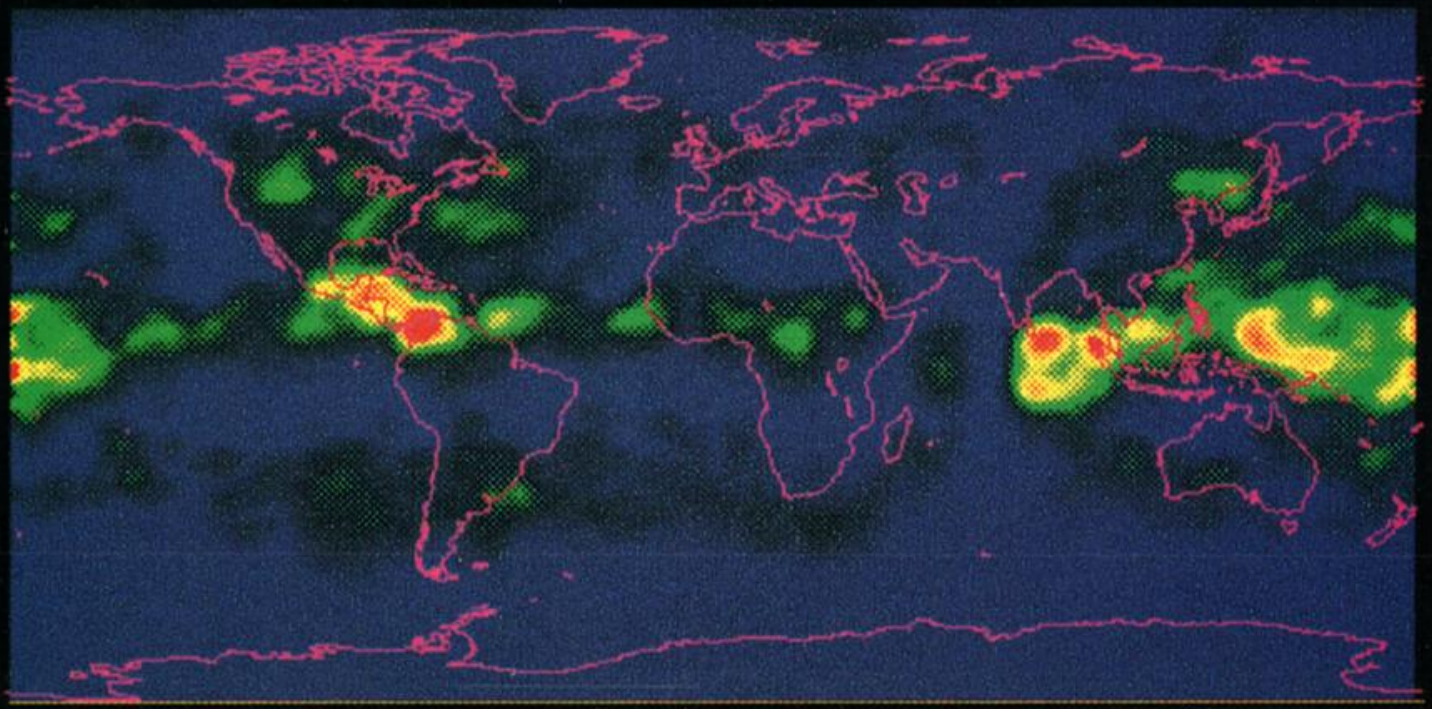

0.0

0. 5

\section{Monthly Mean Optical Depth: Observed (ISCCP)}

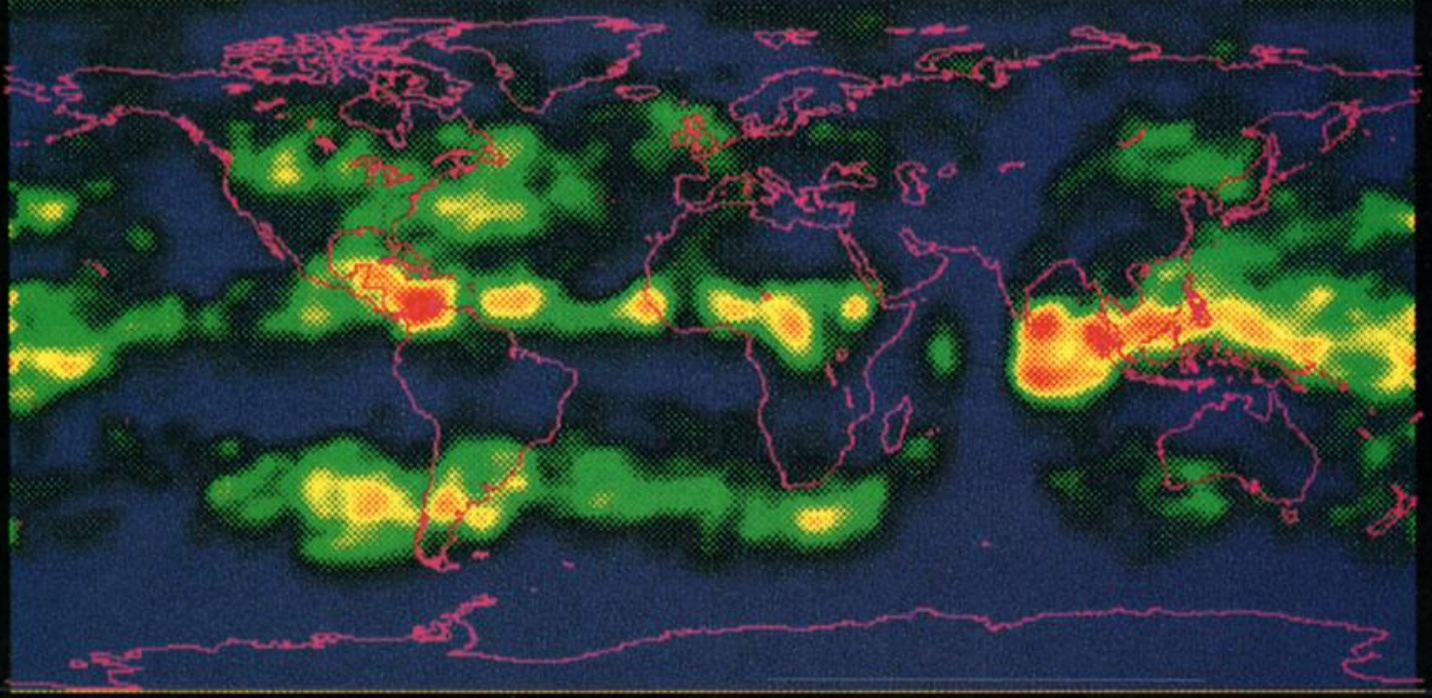

0.0

0. 5

1.0

Plate 1. Monthly mean optical depth from the HD parameterization (top) and International Satellite Cloud Climatology Project (ISCCP) observations (bottom) for July 1987.

This averaging procedure removes the effect of prescribing the same daily occurrence of cirrus for both the observations and the parameterization and provides a clearer picture of the parameterization's ability to cap- ture the observed geographic patterns of cirrus optical thickness.

The geographic distribution of conditionally averaged optical depth is shown in Plate 2. The scale ranges from 0 (blue) 


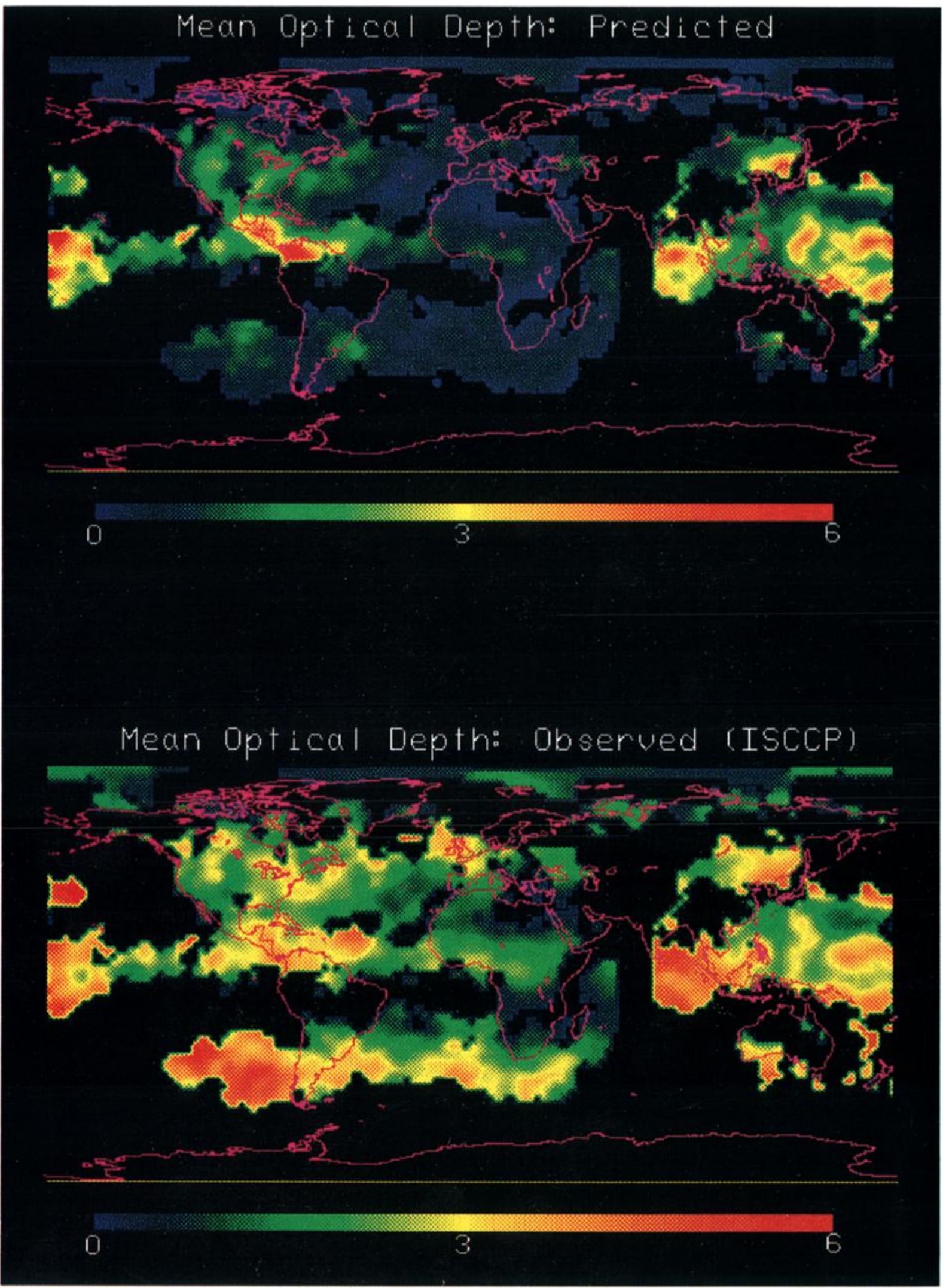

Plate 2. Monthly mean, conditionally averaged optical depth from the HD parameterization (top) and ISCCP observations (bottom) for July 1987.

to 6 (red). The larger values of optical depth in Plate 2 relative to Plate 1 are a consequence of averaging the optical depths from cloudy occasions only rather than from all (clear and cloudy) occasions. Black indicates regions where no cirrus clouds with fractional cover greater than $20 \%$ were observed or the cirrus which did occur were associated with deep convective cloud cover. The absence of cirrus occurs primarily over portions of the northern and southern hemi- 
sphere midlatitude Pacific Ocean, the southern hemisphere subtropics, central Asia, and the southern hemisphere high latitudes. The meridional swath of missing data positioned near the center of the Indian Ocean reflects the boundaries of the GMS and Meteosat geostationary viewing domains. To the extent that geographic variations in effective radius are small relative to the variations in IWP, the comparison of observed and predicted optical depths can be used to evaluate the ability of the parameterization to capture the geographic variations in IWP. Despite a noticeable amount of zonal asymmetry, the primary features are a maximum in optical thickness along the ITCZ, lower values over the subtropics, and a second band of optically thick cirrus over the midlatitudes of North America, South America, and eastern Asia. Interestingly, the parameterized optical depths are noticeably larger over the northern hemisphere midlatitudes $(\tau \sim 2-3)$ than over the southern hemisphere midlatitudes $(\tau \sim 1-2)$, suggesting a difference in cirrus formation processes between these two regions. The observed distribution (bottom) also exhibits a band of thick cirrus over the tropics with optical depths similar in magnitude to those predicted. However, in contrast to the predicted values, the observations also demonstrate equally large cirrus optical depths over the midlatitudes. The discrepancy between the observed and the predicted optical depths is particularly evident over the southern hemisphere midlatitudes where the observed values are frequently a factor of 2 larger than those predicted. This suggests that (1) the parameterization has a tendency to underestimate the IWP over the southern hemisphere midlatitudes relative to other regions; (2) the accuracy of the input parameters (e.g., $w, T$ ) is systematically poorer over the midlatitudes of the southern hemisphere relative to the northern hemisphere; (3) there is a systematic decrease in effective radius away from the tropics toward higher latitudes; or (4) there is an overestimate in the ISCCP retrieved optical depths for the southern hemisphere midlatitudes relative to other regions, perhaps due to systematic differences in cirrus microphysical or macrophysical properties (e.g., particle shape, multilayered clouds).

Despite the differences in magnitude between some of the observed and predicted optical depths the results of this comparison are encouraging in that many geographic features evident in the observed optical thickness distribution are captured by the parameterization. For example, both distributions exhibit a maxima in optical thickness along the Central American isthmus which then extends across the northern tip of South America, merging with a slightly weaker band which stretches from the west coast of Africa into the continental interior. A region of large optical depths located over the central Pacific is also captured by the parameterization. Both the observations and the parameterization exhibit optically thick clouds $(\tau>3)$ just west of Indonesia and in three distinct bands over the western Pacific with an area of optically thin clouds $(\tau<3)$ to the north. Indeed, the close correspondence between the predicted and the observed patterns is particularly impressive over the western Pacific where the spatial features of the distributions are strikingly similar. Over North America the parameterization simulates optically thick clouds in bands along the Rocky and Appalachian mountain ranges with much thinner clouds over the central plains. Over the north Atlantic a band of thick cirrus to the west with much thinner clouds to the east is predicted. These features closely resemble those present in the observations. Recall that these

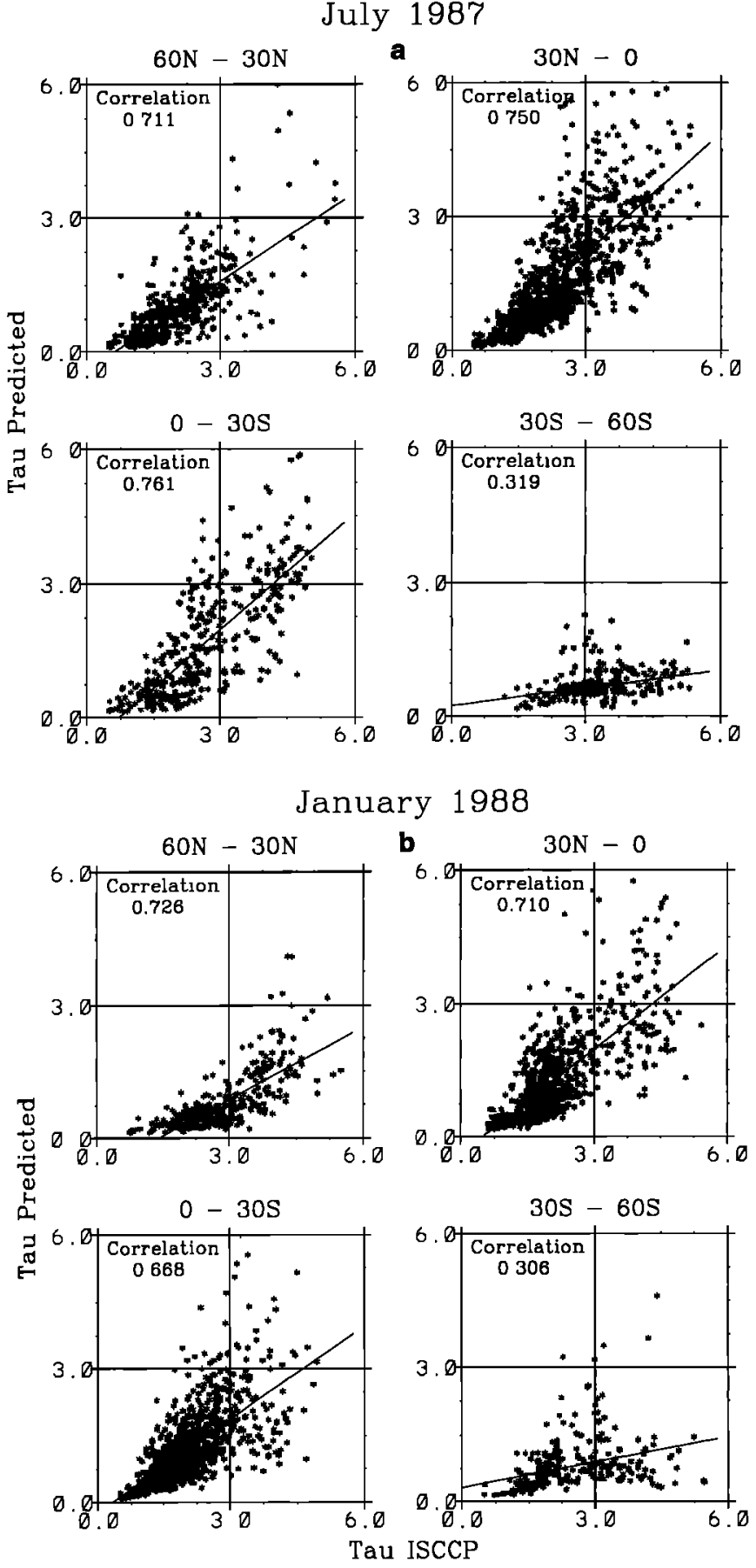

Figure 3. Scatterplots of the ISCCP observed versus predicted optical depth for the HD parameterization for (a) July 1987 and (b) January 1988. Plots are shown separately for selected latitude belts.

values represent averages from cloudy (cirrus) regions only, therefore the spatial agreement is not an artifact of the prescribed occurrence of cirrus. Rather the spatial agreement stems from the ability of the parameterization to yield the observed patterns of optical thickness based upon the atmospheric variables $(T, w, \Gamma, P)$ derived from the cirrus layer.

Figure 3a illustrates scatter plots of the predicted (conditionally averaged) optical depth versus the observed (conditionally averaged) optical depth. To highlight regional depen- 
Table 1. Conditionally Averaged, Predicted Optical Depth Binned Daily According to the Corresponding Observed Optical Depth Category

\begin{tabular}{lccccc}
\hline & Global & $60^{\circ}-30^{\circ} \mathrm{N}$ & $30^{\circ} \mathrm{N}-0^{\circ}$ & $0^{\circ}-30^{\circ} \mathrm{S}$ & $30^{\circ}-60^{\circ} \mathrm{S}$ \\
\hline Thin (0.2-1.3) & 0.55 & 0.51 & 0.62 & 0.52 & 0.49 \\
Moderate (1.3-3.6) & 1.92 & 1.62 & 2.30 & 1.71 & 0.96 \\
Thick (3.6-9.4) & 3.16 & 3.02 & 4.02 & 2.89 & 1.56 \\
\hline
\end{tabular}

Values are shown separately for selected latitude belts.

dencies, plots are shown separately for four latitude belts: $60^{\circ}-30^{\circ} \mathrm{N}, 30^{\circ}-0^{\circ} \mathrm{N}, 0^{\circ}-30^{\circ} \mathrm{S}$, and $30^{\circ}-60^{\circ} \mathrm{S}$. For each of the four regions the spatial correlations are printed in the top left-hand corner. Tropical regions show the highest spatial agreement. Correlations are typically greater than 0.7 , indicating that the parameterization explains over $50 \%$ of the observed spatial variations in optical depth. For July the correlations tend to be highest for the tropics and slightly lower for the northern hemisphere midlatitudes. However, for the southern hemisphere midlatitudes the spatial correlation is very low $(r \sim 0.3)$, indicating that the parameterization explains only about $10 \%$ of the observed spatial variations. The much poorer agreement for the southern hemisphere midlatitudes suggests that additional processes not accounted for in the parameterization are important in determining the geographic patterns of cirrus optical thickness for this region. One possibility is that enhanced baroclinicity over the winter hemisphere midlatitudes may degrade the performance of the parameterization. To investigate this hypothesis, a similar comparison is performed for January 1988 (Figure 3b). As for the July comparison, ECMWF analyses and ISCCP measurements are used to provide the input parameters and cirrus observations. Interestingly, the results for January are very similar to those noted for July. Large correlations $(r \sim 0.7)$ are found over the tropics and northern hemisphere midlatitudes, while poor correlations ( $r$ $\sim 0.3$ ) are noted for the southern hemisphere midlatitudes. This indicates that seasonal effects are not responsible for the poor correlations in the southern hemisphere. Further analysis of the poor performance in the southern hemisphere midlatitudes is provided in section 4.6 where the physical processes controlling the parameterized IWP are examined.

Some insight into the ability of the parameterization to capture the daily values of optical depth is provided in Table 1 , which examines the predicted optical depth corresponding to three broad categories of the observed optical depth: thin $(0.2<\tau<1.27)$, moderate $(1.27<\tau<3.55)$, or thick $(3.55<\tau<9.38)$. To calculate these values, the predicted optical depths are binned into one of the three categories based upon the corresponding observed optical depth for each day and grid box. The values in each bin are then averaged to yield the quantities in Table 1 . The results shown here are for July 1987 only. To examine regional dependencies, the spatial averaging was done both globally and for selected latitude belts. The results illustrate a clear trend of increasing predicted optical depth as the observed optical depth category progresses from thin to moderate to thick. This pattern is evident both globally and for all four latitude belts. Since the categorization was performed on a daily basis, this provides a crude indication of the parameterization's ability to capture temporal variations in optical depth as well as geographic variations. The numbers also illustrate that with the general exception of the southern hemisphere midlatitudes the mean predicted optical depths lie near the central value of the observed category for the thin and moderate cirrus. However, for thick cirrus the predicted values tend to be systematically underestimated. The greater discrepancy for thicker cirrus may stem from difficulties in excluding cirrus associated with convective systems in the observed optical depths. Over the southern hemisphere midlatitudes the optical depths are systematically lower for all three categories, again revealing a latitudinal dependence in the discrepancies between the observed and the parameterized optical depths.

\subsection{Cirrus Emissivity}

To examine the optical properties of cirrus in the infrared (IR) spectral region, Plate 3 illustrates a map of the predicted monthly mean cirrus emissivity for July. The scale ranges from 0 (blue) to 1 (red). The predicted emissivity is approximated as $\varepsilon=1-e^{-\tau}$, where $\tau$ is the IR optical depth determined from the parameterized IWP using a $\bar{Q}_{\lambda}$ calculated for ice spheres at $\lambda=15 \mu \mathrm{m}$ (see section 2). To evaluate the parameterization, observations of effective emissivity for clouds above 500 mbar are overlain as contours in Plate 3. The effective emissivity is calculated from GOES-VAS observations for the period July 1-31, 1987, using the $\mathrm{CO}_{2}$-slicing technique [Wylie and Menzel, 1989]. This technique uses IR spectral observations in the $\mathrm{CO}_{2}$ vibration band to derive cloud top pressures. A linear dependence of the radiance upon cloud amount is then used to derive an effective emissivity for the cloud. The ability of the $\mathrm{CO}_{2}$-slicing technique to identify partially transmissive clouds makes it particularly useful for observing cirrus which are frequently nonopaque and hence often go undetected or are categorized as opaque clouds at lower levels. The observed effective emissivities represent the product of cloud amount and emissivity. Since the variations in cloud amount are not included in the parameterization, this figure provides only a qualitative comparison of IR properties of cirrus clouds. It is noted, however, that most of the variations in effective emissivity are due to changes in cloud emissivity rather than cloud amount (W. P. Menzel, personal communication, 1994). Although there are some differences in detail between the distributions, generally speaking both the observed and the predicted emissivities exhibit very similar patterns. The chief features in both the observed and the predicted distributions are a minima over the west coast of the U.S. followed by a maxima extending along the eastward flank of the Rocky Mountains. Continuing eastward, a distinct minima over the central plains is observed followed by maxima which stretches along the Appalachians and then extends off east coast into the North Atlantic. Although quantitative statements regarding the accuracy of the parameterized emissivities are difficult due to uncertainties surrounding both the effective radius and the actual cloud amount, the qualitative agreement between the spatial patterns of the predicted and observed cirrus IR emissivity is encouraging and is consistent with the good spatial agreement noted for visible optical depths (sections 4.2-4.3).

\subsection{Required Effective Radius}

The comparison of the conditionally averaged optical depth clearly reveals regions of significantly differing magnitudes between the observed and the predicted optical 
depths. However, as discussed previously, the relationship between optical depth and IWP depends upon the effective radius $\left(r_{e}\right)$ for which there are a wide range of estimates. A study by Prabhakara et al. [1988], using split-window infrared measurements of the upwelling radiation between 10 and $12 \mu \mathrm{m}$, derived estimates of $r_{e}$ ranging from 5 to $10 \mu \mathrm{m}$. In comparison, direct measurements using airplane cloud probes provide estimates which are typically in excess of 100 $\mu \mathrm{m}$ [Heymsfield et al., 1990]. Indeed, estimates of $r_{e}$ taken from the same cirrus formation during the First ISCCP Regional Experiment (FIRE) Cirrus Intensive Field Observation [Starr and Wylie, 1990] exhibit a wide range of $r_{e}$ depending upon the measurement technique used. Estimates of $r_{e}$ based upon the split window technique varied from 12-24 $\mu \mathrm{m}$ [Spinhirne and Hart, 1990] to 20-40 $\mu \mathrm{m}$ [Ackerman et al., 1990], while the value estimated from observations of the reflected radiation in the near-infrared spectrum was $60 \mu \mathrm{m}$ [Wielecki et al., 1990] and the value derived from airplane probe measurements was $200 \mu \mathrm{m}$ [Wielecki et al., 1990]. The markedly larger values of $r_{e}$ obtained from airplane probe measurements likely reflects a sampling bias since few probes are capable of accurately detecting ice particles smaller than approximately $20 \mu \mathrm{m}$ [Heymsfield and Platt, 1984; Wielecki et al., 1990].

Given the present uncertainty surrounding the size distribution of cirrus particles, it is insightful to examine the geographic distribution of $r_{e}$ required for the parameterized IWP to produce the observed distribution of optical depth, $r_{e}=3 \bar{Q}_{\lambda}$ IWP $/ 4 \tau \rho_{\text {ice }}$, where IWP is that predicted by the parameterization and $\tau$ is the corresponding ISCCP optical depth. The results for July, shown in Plate 4, illustrate large geographic variations in the "required" $r_{e}$. Although the global mean $r_{e} \sim 7 \mu \mathrm{m}$ is somewhat lower than most estimated values, roughly $80 \%$ of the required $r_{e}$ between $60^{\circ} \mathrm{N}$ and $30^{\circ} \mathrm{S}$ lie between 5 and $40 \mu \mathrm{m}$, which is the range derived from the aforementioned split-window techniques. The largest $r_{e}(10-25 \mu \mathrm{m})$ occur primarily over the central and western Pacific Ocean, Central America and northern South America. The required $r_{e}$ tends to decrease from the tropics toward higher latitudes, with typical values over the northern hemisphere midlatitudes ranging from 5 to $15 \mu \mathrm{m}$. However, markedly lower $r_{e}(<5 \mu \mathrm{m})$ are required to explain the differences over the southern hemisphere midlatitudes. This value of $r_{e}$ is considerably lower than most of the above listed estimates, suggesting that the predicted IWP for these regions is too small or that the observed optical depths are too large. Similar results are noted for January (not shown). Clearly, further research is needed to better ascertain the actual values of $r_{e}$ and their geographic and vertical variations. Global estimates of $r_{e}$ determined via the split window technique using IR radiances from polarorbiting satellites would provide useful estimates of the spatial and temporal variability in $r_{e}$.

\subsection{Physical Interpretation of the Results}

Despite the uncertainty surrounding the appropriate effective radius of ice particles, results from section 4.2 demonstrate the ability of the parameterization to capture many of the observed geographic variations in cirrus optical thickness. This section analyzes the functional dependence of the parameterization to determine which variables are most responsible for the good spatial agreement. Of the four input variables used in the parameterization $(T, w, P, \Gamma)$, the parameterized IWP is primarily sensitive to temperature. This is demonstrated in Figure 8a which shows a set of curves depicting the predicted IWP as a function of temperature for four different values of vertical velocity $(0.01,0.05$, $0.2,0.5 \mathrm{~m} / \mathrm{s}$ ), a fixed pressure (300 mbar), and a fixed lapse rate $(\Gamma=0.007 \mathrm{~K} / \mathrm{m})$. Temperature is the principal governing variable, with the IWP varying by up to 3 orders of magnitude throughout the observed range of $T$. At warm temperatures, vertical velocity also becomes an important factor, however for $T<225 \mathrm{~K}$, the predicted IWP is less than $0.01 \mathrm{~g} / \mathrm{m}^{2}$ regardless of the vertical velocity. A set of curves depicting the IWP $-T$ relationship for various lapse rates and a fixed pressure and vertical velocity is shown in Figure $4 \mathrm{~b}$. This diagram demonstrates that lapse rate is of much less importance in determining IWP than either temperature or vertical velocity.

The strong dependence of the parameterized IWP upon temperature is confirmed in Figure 5 which illustrates a scatterplot of the conditionally averaged optical depth versus the corresponding average of the cloud layer temperature. The cloud layer temperature is the temperature which is input into the HD parameterization from the ECMWF analyses. This plot displays a clear increase in optical depth with increasing temperature. However, the relationship depicted in Figure 5 is clearly nonlinear. Physically, the nonlinear increase in IWP with temperature stems from the exponential dependence of the saturated water vapor content upon temperature dictated by the Clausius Clapeyron relationship. This is demonstrated by the dashed curve in Figure 5 which shows the expected rate of increase of optical depth with temperature, if it increased at the same rate as the saturation vapor pressure of water. This curve captures much of the nonlinear rise on the predicted optical depth illustrated in Figure 5.

Several observational studies [Platt and Harshvardan, 1988; Heymsfield and Platt, 1984] have also demonstrated a strong dependence of cirrus IWP upon atmospheric temperature. This dependence is also evident in the ISCCP observations. Figure 6a shows a scatterplot of the conditionally averaged ISCCP optical depth versus the corresponding cloud layer temperature (from ECMWF) using all data points between $60^{\circ} \mathrm{N}$ and $30^{\circ} \mathrm{S}$ latitude for July. An increase in optical thickness with increasing temperature is evident with a correlation of 0.771 between the two variables. The observed relationship is also much more linear than that noted for the predicted optical depth. A possible explanation for this linearity could be a systematic increase in effective radius with temperature as has been noted in previous observational studies [Platt and Harshvardan, 1988; Heymsfield and Platt, 1984]. This would reduce the rate of increase of optical depth with temperature, resulting in a more linear relationship. When data from the southern hemisphere midlatitudes, $30^{\circ}-60^{\circ} \mathrm{S}$, are included in the plot (Figure 6b), the scatter increases markedly. To further illustrate this point, the data in Figure $6 \mathrm{~b}$ are categorized into two groups: (1) $60^{\circ} \mathrm{N}$ to $30^{\circ} \mathrm{S}$, denoted by "dots" and (2) $30^{\circ}-60^{\circ} \mathrm{S}$, denoted by "asterisks." This plot reveals that for a given temperature the optical depth from $30^{\circ}$ to $60^{\circ} \mathrm{S}$ are up to a factor of 2 larger than those from the other regions. This indicates that the optical depth-temperature relation observed for the tropics and northern hemisphere midlatitudes breaks down over the southern hemisphere midlatitudes, suggesting a fundamental difference in the processes which 


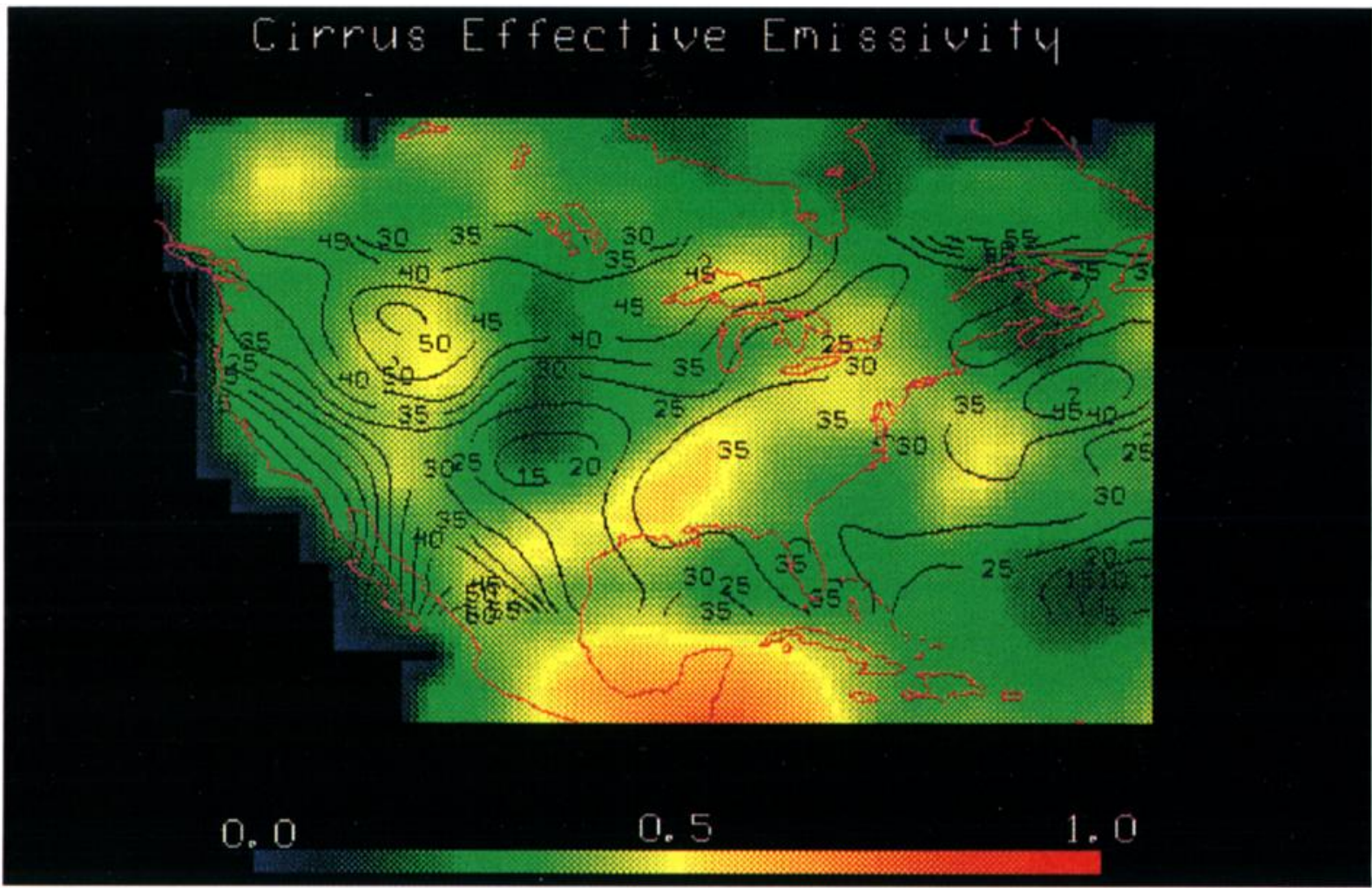

Plate 3. Map of the predicted emissivity from the HD parameterization overlain by contours of effective emissivity from the $\mathrm{CO}_{2}$-slicing technique.

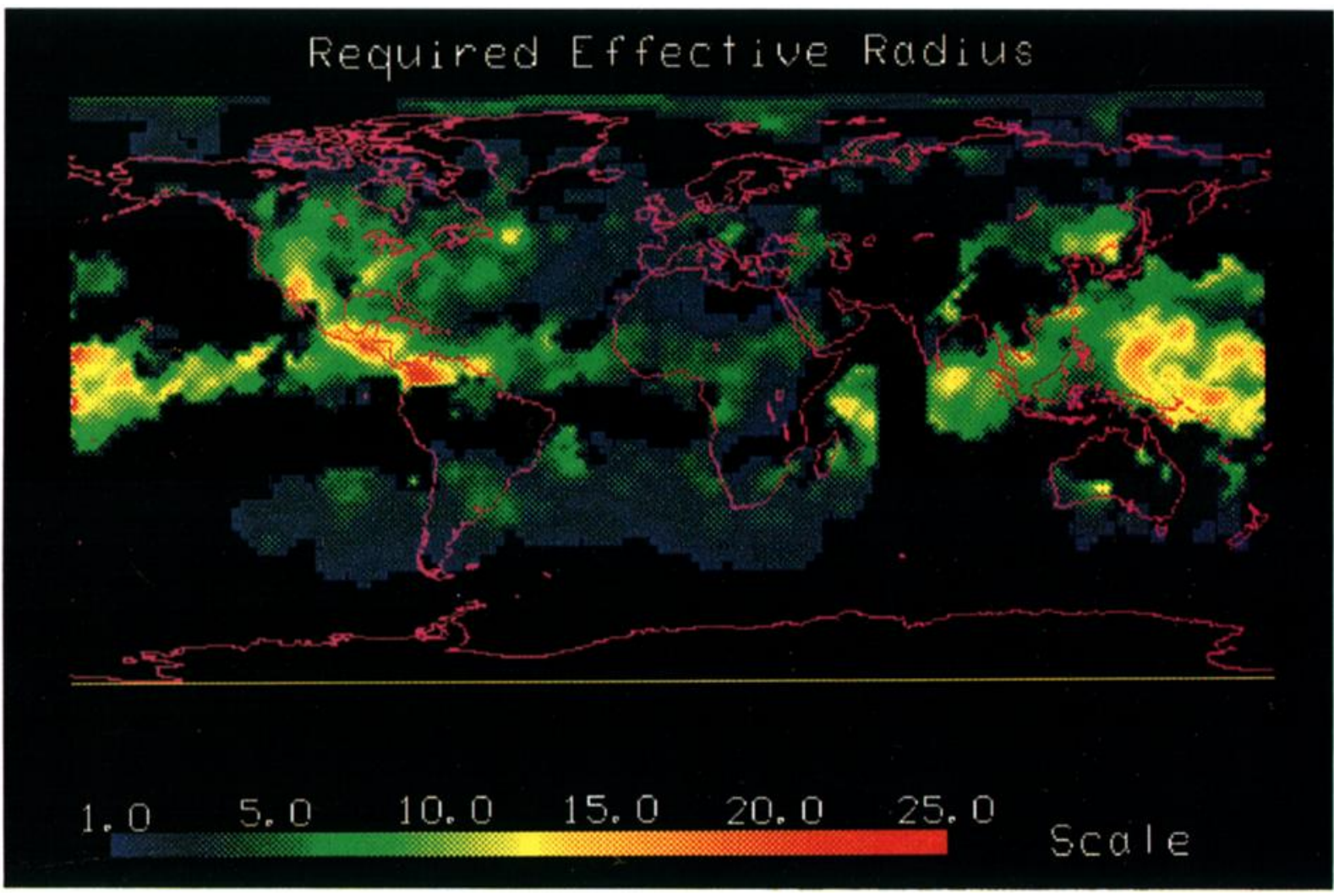

Plate 4. Map of the effective radius required for the predicted IWP to yield the observed ISCCP optical depth for July 1987. 
determine optical thickness for that region. A similar pattern is evident in January (not shown).

The latitudinal dependence of the observed optical depthtemperature relationship is further demonstrated in Figure 7. This figure depicts the zonally averaged, predicted and observed optical depth (Figure 7a) and the zonally averaged, cloud layer temperature and pressure (Figure $7 \mathrm{~b}$ ) for July. These curves indicate that the lower values of predicted optical depth in the midlatitudes relative to those in the tropics are largely attributable to the colder cloud layer temperatures at the higher latitudes. The predicted optical depths also are lower in the southern hemisphere midlatitudes relative to the northern hemisphere midlatitudes in agreement with the temperature differences between the two hemispheres. However, the observed optical depth does not exhibit a similar correlation with latitudinal variations in temperature. Indeed, even though the cloud layer temperature decreases by roughly $4 \mathrm{~K}$ from the $45^{\circ} \mathrm{N}$ to $45^{\circ} \mathrm{S}$, the observed optical depth is actually larger in the southern hemisphere midlatitudes than in the northern hemisphere midlatitudes.

The following possible causes of the large discrepancies between the predicted and the optical depths in the southern hemisphere are presented.

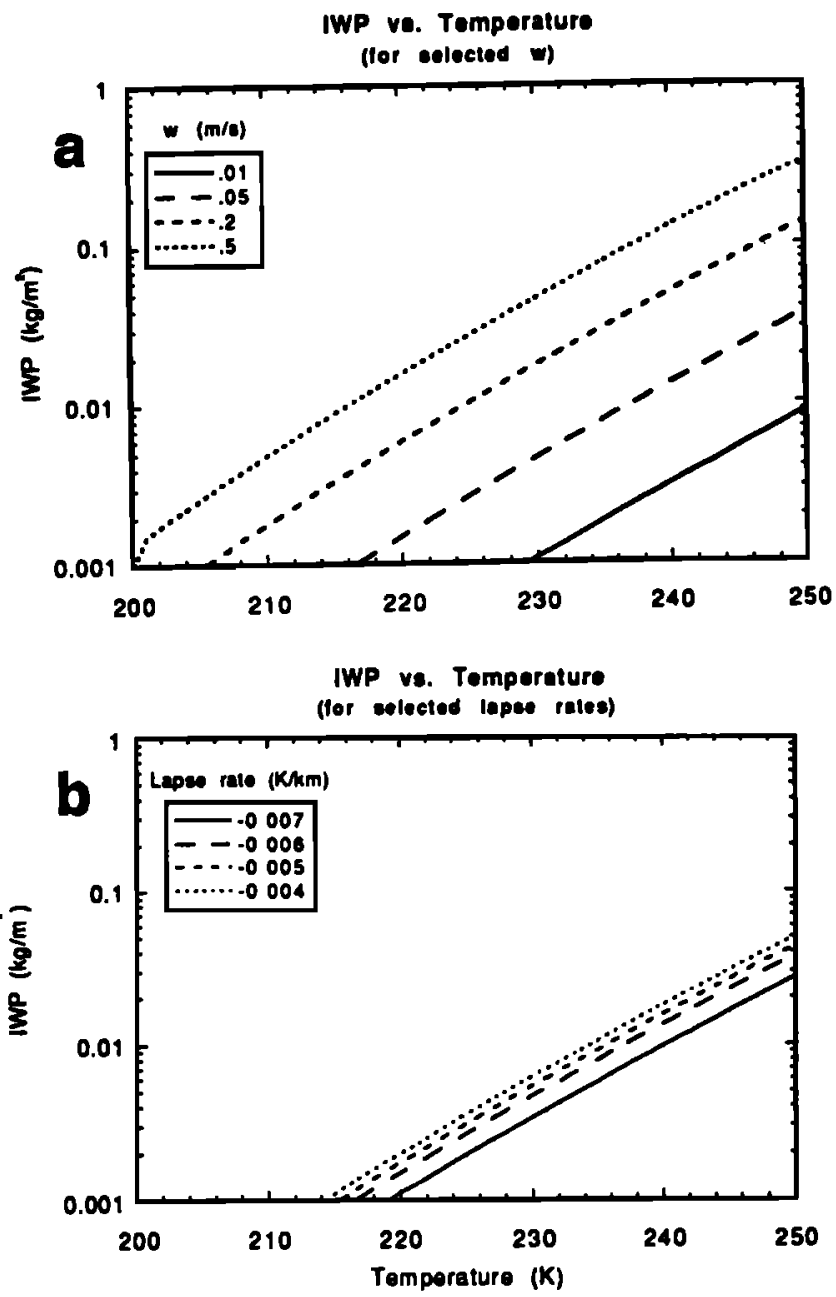

Figure 4. Curves depicting the predicted IWP as a function of temperature for (a) four different vertical velocities and (b) four different lapse rates.

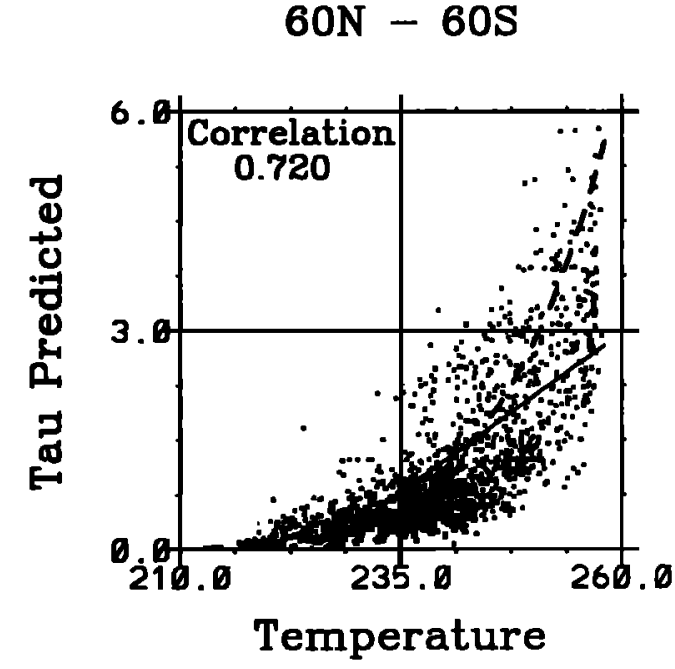

Figure 5. Scatterplot of the conditionally averaged optical depth predicted by the HD parameterization versus the corresponding cloud layer temperature. Data are for July 1987.

Errors in the analyses. The input parameters, particularly temperature and vertical velocity, may be of consistently poorer quality over the southern hemisphere midlatitudes, where conventional observations are scarce, relative to the northern hemisphere midlatitudes [see Trenberth and Olson, 1988].

Parameterization deficiencies. Additional mechanisms not explicitly accounted for in the parameterization may be important in determining the ice water path for the southern hemisphere midlatitudes. Since the parameterization was developed from a relatively small sample of observations with limited geographic coverage, all relevant processes may not be adequately represented.

Subgrid variability. Subgrid variations in either the fractional coverage of cirrus cloud or the input parameters (e.g., vertical velocity) could results in significant discrepancies since the parameterization considers all quantities to represent spatial averages over the entire $2.5^{\circ} \times 2.5^{\circ}$ grid box.

Microphysical variations. The effective radius may be smaller over the southern hemisphere midlatitudes relative to other regions, resulting in systematically larger observed optical depths for the same IWP. However, this effect would not explain the poorer spatial correlations noted for the southern hemisphere midlatitudes.

Errors in the ISCCP retrieval. Systematically larger errors in the ISCCP retrievals of cirrus thickness for the southern midlatitudes could contribute to the discrepancies, however there is no obvious reason to suspect this.

\section{Summary}

Satellite observations of cloud optical properties are combined with global analyses of the temperature, moisture, and wind distribution to evaluate the ability of the HD parameterization to predict the ice water path of cirrus clouds. ECMWF analyses are utilized to reduce uncertainties in the input variables for the parameterization. This helps to isolate errors in the predicted cloud properties due to deficiencies in the input parameters from those due to errors in the param- 

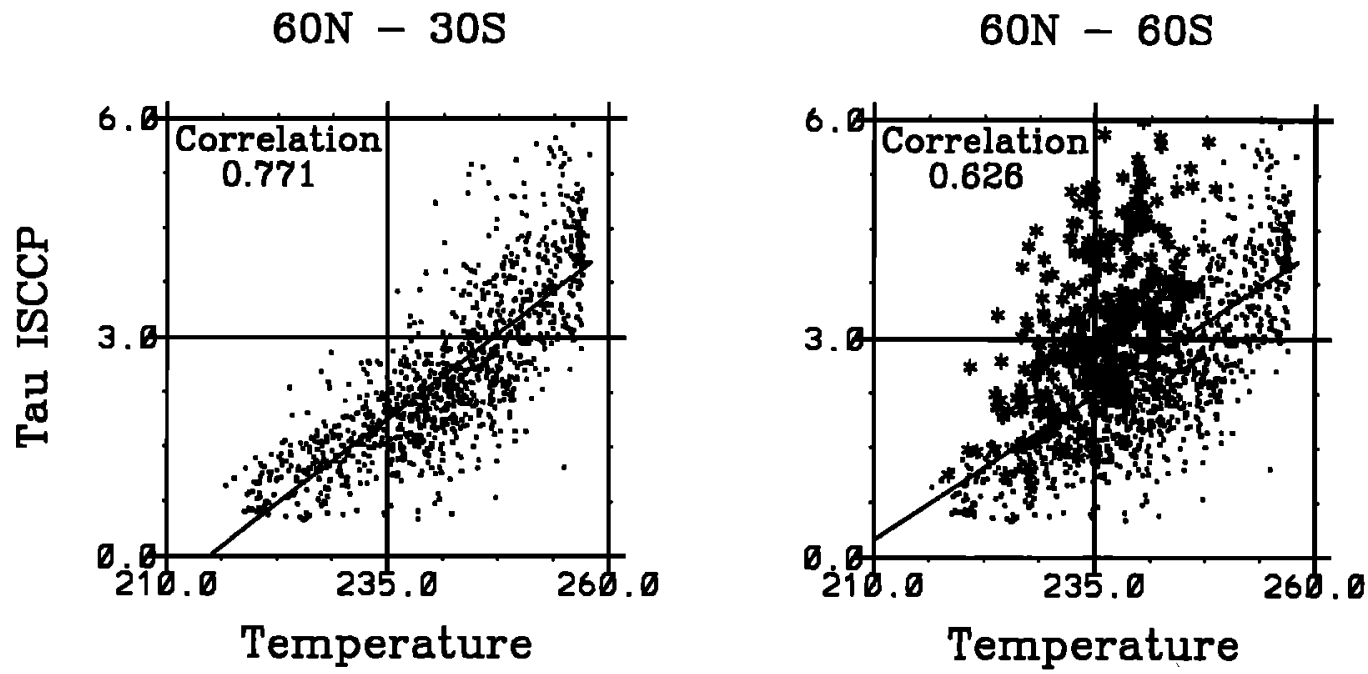

Figure 6. Scatterplot of the conditionally averaged optical depth observed by ISCCP versus the corresponding cloud layer temperature: (a) $60^{\circ} \mathrm{N}$ to $30^{\circ} \mathrm{S}$ and (b) $60^{\circ} \mathrm{N}$ to $60^{\circ} \mathrm{S}$. Data are for July 1987.

eterization itself. To eliminate the uncertainty in predicting cirrus formation, the occurrence of cirrus is prescribed using ISCCP satellite observations, while the ice water path is calculated based upon the corresponding dynamic and thermodynamic variables for that grid box from ECMWF. This enables the evaluation to focus strictly on the parameteriza-
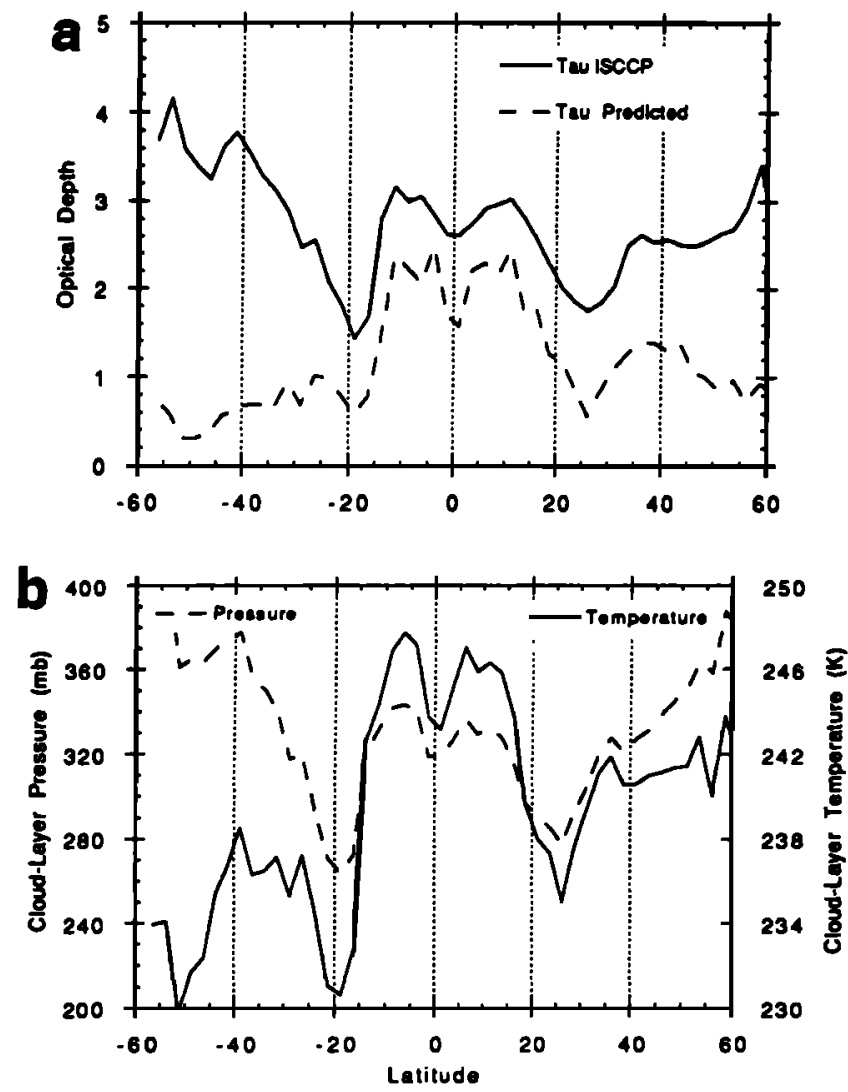

Figure 7. Zonally averaged optical depth from (a) ISCCP observations and the HD parameterization and (b) the corresponding cloud layer temperature and pressure. Data are for July 1987. tion of cirrus optical properties and not on the parameterization of cirrus occurrence. Although this investigation focuses on a specific cloud parameterization, it also serves a more general purpose of outlining the utility of combining operational analyses and satellite observations for evaluating cloud parameterizations in general.

Overall, the HD parameterization is quite successful in capturing many of the observed spatial patterns of cirrus optical properties. This is true when compared with both visible optical depths and IR effective cloud emissivities. Spatial correlations between the observed and the predicted visible optical depths are typically greater than 0.7 for the tropics and midlatitudes. Much of the success of the parameterization stems from the strong temperature dependence evident in both the predicted and the observed optical properties which is largely attributable to the exponential dependence of the saturation vapor pressure of water on temperature. However, for much of the southern hemisphere storm tracks, both the predicted IWP and spatial agreement of the parameterization are systematically lower. This suggests that other mechanisms in addition to temperature are important in determining the optical thickness for these regions and that these mechanisms either are not represented accurately in the ECMWF analysis or are not modeled accurately in the parameterization. Additional factors such as systematic geographic variations in effective radius or errors in the optical-depth retrieval could also contribute to the discrepancy. Despite the qualitative success of the parameterization in reproducing the observed geographic patterns of cirrus optical depth, a more quantitative assessment of the parameterization is not possible due to uncertainties in the effective radius of cirrus particles. Present estimates of $r_{e}$ range over an order of magnitude. From a cloud-parameterization perspective this uncertainty translates into an equally large range of uncertainty in determining the optical depth from model predictions of IWP. Thus it is difficult to evaluate the significance of the differences between the observed and the predicted optical depths until more reliable estimates of cirrus effective radius become available. This poses problems not only for future 
evaluation studies but also for the implementation of physically based cirrus parameterizations in GCMs and therefore represents an area where future observations are needed.

Acknowledgments. We thank S. A. Ackerman, N. C. Lau, W. P. Menzel, V. Ramaswamy, W. B. Rossow, and two anonymous reviewers for constructive comments and suggestions; Don Wylie for providing the $\mathrm{CO}_{2}$-slicing cloud emissivity measurements; and the NCAR Data Support Section for providing the ECMWF analyses and ISCCP observations. A portion of this study was completed with support from a NASA Global Change Fellowship to B.J.S. and NASA grant NAG5-1056 TO L.J.D.

\section{References}

Ackerman, S. A., and G. L. Stephens, The absorption of solar radiation by cloud droplets: An application of anomalous diffraction theory, J. Atmos. Sci., 44, 1564-1588, 1987.

Ackerman, S. A., W. L. Smith, J. D. Spinhirne, and H. E. Revercomb, The 27-28 October 1986 FIRE cirrus case study: Spectral properties of cirrus clouds in the 11-12 $\mu \mathrm{m}$ window, Mon. Weather Rev., 118, 1990.

Cess, R. D., et al., Intercomparison and interpretation of climate feedback processes in 19 atmospheric general circulation models, J. Geophys. Res., 95, 16,601-16,615, 1990.

Donner, L. J., Radiative forcing by parameterized cirrus clouds in a GCM, Preprints presented at the 8th Conference on Atmospheric Radiation, Am. Meteorol. Soc., Nashville, Tenn., 1994. (Available from the American Meteorological Society, Boston, Mass., 1994.)

Ebert, E. E., and J. A. Curry, A parameterization of ice cloud optical properties for climate models, J. Geophys. Res., 97, 3831-3836, 1992.

Heymsfield, A. J., and C. M. R. Platt, A parameterization of the particle size spectrum of ice clouds in terms of the ambient air temperature and ice water content, J. Atmos. Sci., 41, 846-855, 1984.

Heymsfield, A. J., and L. J. Donner, A scheme for parameterizing ice-cloud water content in general circulation models, J. Atmos. Sci., 47, 1865-1877, 1990.

Heymsfield, A. J., K. M. Miller, and J. D. Spinhirne, The 27-28 October 1986 FIRE IFO cirrus case study: Cloud microstructure, Mon. Weather Rev., 118, 2313-2328, 1990.

Kiehl, J. T., and V. Ramanathan, Comparison of cloud forcing derived from the ERBE with that from the NCAR CCM, $J$. Geophys. Res., 95, 11,679-11,698, 1989.

Menzel, W. P., D. P. Wylie, and K. I. Strabala, Seasonal and diurnal changes in cirrus clouds as seen in four years of observations with the VAS, J. Appl. Meteorol., 31, 371-385, 1992.

Minnis, P. D., F. Young, K. Sassen, J. M. Alvarez, and C. J. Grund, The 27-28 October FIRE IFO cirrus case study: Cirrus parameter relationships derived from satellite and lidar data, Mon. Weather Rev., 118, 2402-2425, 1990.

Platt, C. M. R., and Harshvardan, The temperature dependence of cirrus extinction: Implications for climate feedback, J. Geophys. Res., 93, 11,051-11,062, 1988.

Prabhakara, C., R. S. Fraser, G. Dalu, M. C. Wu, and R. J. Curran,
Thin cirrus clouds: Seasonal distribution over oceans deduced from Nimbus-4 IRIS, J. Appl. Meteorol., 27, 379-399, 1988.

Randall, D. A., Harshvardan, D. A. Dazlich, and T. G. Corsetti, Interactions among radiation, convection and large-scale dynamics in a general circulation model, J. Atmos. Sci., 46, 1943-1970, 1989.

Ramanathan, V., E. J. Pitcher, R. C. Malibe, and M. L. Blackmon, The response of a spectral general circulation model to refinements in the radiative processes, J. Atmos. Sci., 40, 605-630, 1983.

Rossow, W. B., L. C. Garder, and P. Li, International Satellite Cloud Climatology Project (ISCCP) documentation of cloud data, WMO Rep., WMO/TD-266, 43 pp., World Meteorol. Organ., Geneva, 1988.

Shukla, J., and Y. Sud, Effects of cloud-radiation feedback on the climate of a general circulation model, J. Atmos. Sci., 38, 23372353, 1981.

Smith, L. D., and T. H. Von der Haar, Cloud-radiation interactions in a general circulation model: Impact upon the planetary radiation balance, J. Geophys. Res., 96, 893-913, 1991.

Soden, B. J., Validation of cloud forcing simulated by the NCAR Community Climate Model using observations from the ERBE, $J$. Geophys. Res., 97, 18,137-18,159, 1992.

Spinhirne, J. D., and W. D. Hart, Cirrus structure and radiative parameters from airborne lidar and spectral radiometer observations: The 28 October 1986 FIRE study, Mon. Weather Rev., 118, 2329-2340, 1990.

Starr, D. O'C., and D. P. Wylie, The 27-28 October 1986 FIRE cirrus case study: Meteorology and clouds, Mon. Weather Rev., $118,2259-2287,1990$.

Stephens, G. L., S. Tsay, P. W. Stackhouse, and P. Flatau, The relevance of microphysical and radiation properties of cirrus clouds to climate and climatic feedback, J. Atmos. Sci., 47, 1742-1753, 1990.

Takano, Y., K. N. Liou, and P. Minnis, The effects of small ice crystals on cirrus infrared radiative properties, J. Atmos. Sci., 49, 1487-1493, 1992.

Tiedtke, M., W. A. Heckley, and J. M. Slingo, Tropical forecasts at ECMWF: On the influence of physical parameterization on the mean structure of forecasts and analyses, $Q$.J.R. Meteorol. Soc., 114, 639-664, 1988.

Trenberth, K. E., and J. G. Olson, An evaluation and intercomparison of global analyses from the National Meteorological Center and the European Centre for Medium Range Weather Forecasts, Bull. Am. Meteorol. Soc., 69, 1047-1057, 1988.

Wielicki, B. A., J. T. Suttles, A. J. Heymsfield, R. M. Welch, J. D. Spinhirne, M. C. Wu, D. O'C. Starr, L. Parker, R. F. Arduini, The 27-28 October FIRE IFO cirrus case study: Comparison of radiative transfer theory with observations by satellite and aircraft, Mon. Weather Rev., 118, 2356-2376, 1990.

Wylie, D. P., and W. P. Menzel, Two years of cloud cover statistics using VAS, J. Clim. Appl. Meteorol., 2, 380-392, 1989.

L. J. Donner and B. J. Soden, NOAA GFDL, Princeton University, P.O. Box 308, Princeton, NJ 08542 .

(Received December 5, 1993; revised April 6, 1994; accepted April 8, 1994.) 